\title{
Research on the Key Technology of Electric Power Safety Production Assessment and Early Warning
}

\author{
Yun $\mathrm{Wu}^{1, \mathrm{a}^{*}}$, Haojie Shu ${ }^{2, \mathrm{~b}}$, Lishan $\mathrm{Bao}^{3, \mathrm{c}}$ and Guannan $\mathrm{Xu}^{4, \mathrm{~d}}$ \\ 1, 2, 4 Northeast Dianli University, Jilin, China \\ ${ }^{3}$ State Grid Jiangsu Electric Power Company Information and Communications Branch, Nanjing \\ Jiangsu, China

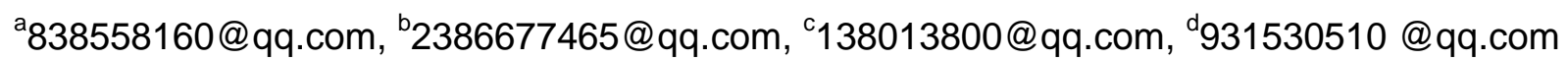

Keywords: Electric power safety production; Data collection; Indicator system; Assessment model

\begin{abstract}
Electric power is the main energy of enterprise production and motivation of the social development. The reliable and safety power supply is quite significant to guarantee the continuity of enterprise production, improvement of the economic efficiency and other aspects of safety production [1]. Currently, most electric power enterprises evaluate safety in terms of electric power production by manual ways. And it is hard to make an effective prediction of potential safety risk. To settle this problem, this paper constructs an integral index system of electric power production assessment and early warning of venture by using the data pre-processing method of combining multiple data resources collection and dynamic storage. The visualization of electric power safety production assessment and early warning will be realized. It is loaded with significance of ensuring electric power safety production, lowering the casualties and providing electric energy.
\end{abstract}

\section{Introduction}

At present, China's economy is in a period of rapid development with electricity production playing a vital role. The safety production of the power system in related to economic development of every aspects of the country, the society and people's daily life [2]. According to the statistics the China Electricity Regulatory Commission, in 2012, a total of 99 electricity security incidents occurred with deaths of 193 people. To estimate roughly, the direct and indirect economic losses may be as high as nearly 100 billion [3]. Therefore, how to improve the safety of electric power production is particularly important. Currently, most of the electric power enterprises make power safety production assessment through artificial way. The efficiency and accuracy of it is questionable, and it is even more difficult to achieve the effective prediction of potential security risk.

Based on the power production safety evaluation methods and risk warning in-depth exploration, this paper explore the power production safety evaluation and early warning methods with cutting-edge information including multi-source data collection, data mining, principal component analysis, information diffusion, GIS technology from perspectives such as theoretical research, key technology and software development. It is of practical significance to the protection of power production safety and reduction of casualties and supply of safe, reliable, qualified energy to users. Power production safety evaluation and of key technologies of early warning is that related with Electricity production safety level and is usually determined by four factors of man, machine and environment management. This article does something of data acquisition, processing, evaluation and early warning from these four aspects. The specific system framework is shown in Figure 1: 


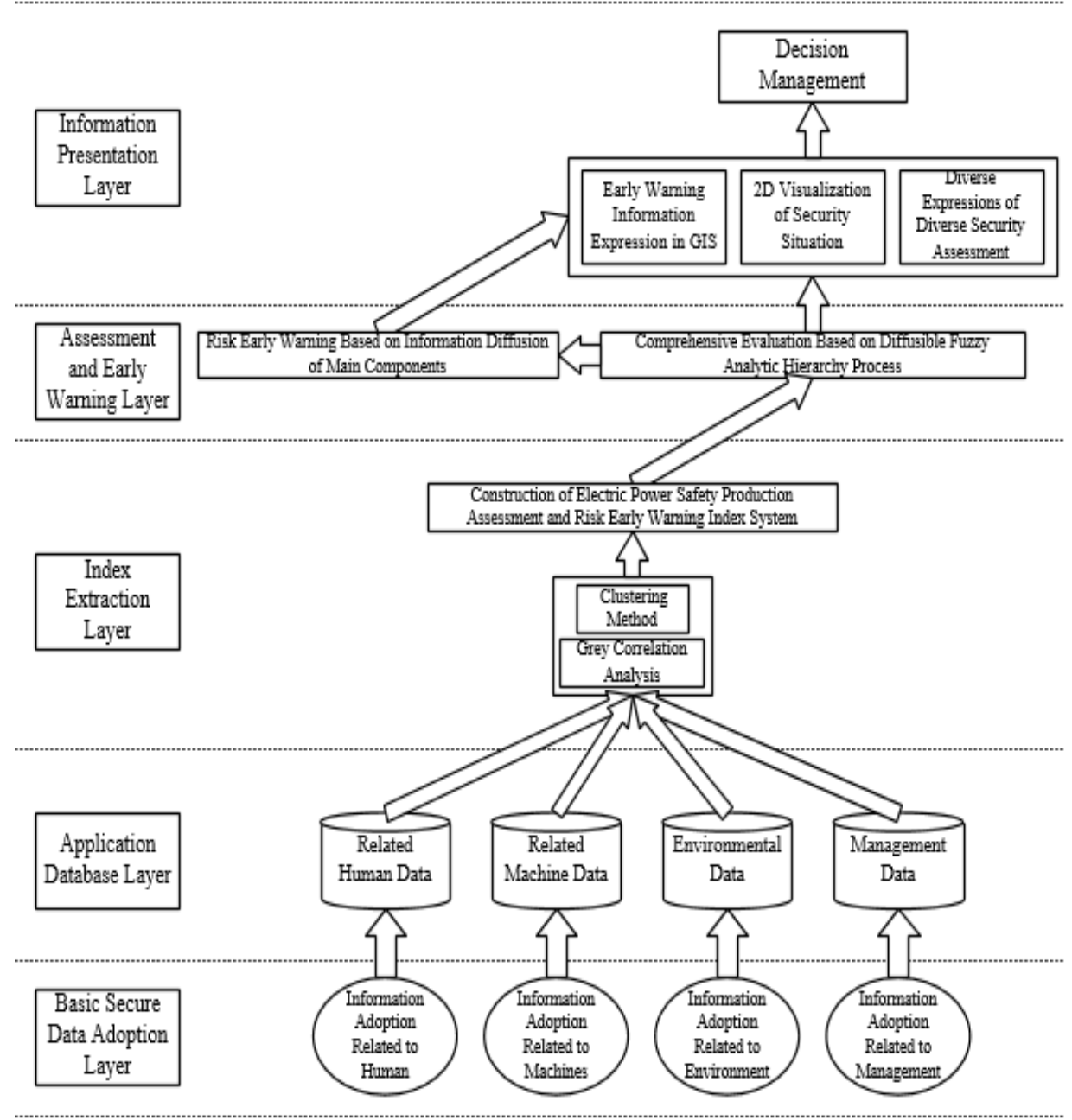

Figure 1. System Architecture

\section{The Data Pre-processing Method Based on the Combination of Multi-source Data Adoption of Service Correlation and Dynamic Storage}

Data adoption is a precondition of electric power safety production assessment. In order to improve the accuracy of electric power safety production assessment, this paper does some data collection coming from four aspects of human , machine, environment and management, including all kinds of equipment operation state, machine hidden danger rectification scheme, plan of staff training, certificate management ,"three assessment" files, contingency plans and security incident reports. The data from multi-system is massive varied (including structured data and unstructured data) and redundant. Based on the fundamental of In-depth research of the current main data collection data adoption methods and aiming at the particularity of the problem of electricity business data collection, this paper makes use of data adoption and processing method based on association business as follows:

(1)The data item corresponding to business with the same acquisition cycle will be sent to a data item collation queue and the heuristic algorithm to the data item queue in sequence will be operated [4].

(2) Complete data adoption with the sequential scheduling of the data items placed in the queue

(3) Make use of the data fusion theory and the combination of machine data classification algorithm of supporting vector machine, extract abstractly in a high level from data information according to the attribute of electric power safety production data, retain the main attributes, filter the redundant information, to achieve the goal of simplify the amount of data and dynamic storage. 


\section{To Make Use of Clustering-grey Correlation Analysis Methods to Construct the Index System of Electric Power Safety Production Assessment and Venture Early Warning}

The electric power safety production assessment is to evaluate the status of electric power production systematically and it is a complex process. There are many factors involved in the assessment process. In order to ensure the representativeness index factor screening results, we need an objective assessment principles and effective method of constructing the index system. However, the existing index system construction method mostly expertise subjective method of constructing the main disadvantage of a strong subjectivity. Therefore, in order to fully and accurately reflect the power production safety level, this paper bases on modern safety management theory for power production safety features, fully consider the human production involved a result, the machine due to environmental factors and management factors, and the use of poly class - gray correlation analysis index system

The main idea is to calculate the size of the degree of association between indicators of computers, and then classify them. To achieve index clustering by the analysis of data samples judging, whether the contact of them is close or not according to the degree of the similarity of sequent linear geometry. The bigger the degree of similarity curve is, the smaller the degree of correlation is. Conversely, the bigger the degree of correlation is[5-6]. The related information of different kinds of details will be shown. The appropriate indicators will be chosen with the setting of the minimum confidence value and the minimum support to achieve the confidence and support associated with different degrees of analysis. Specific human factors, machine due to environmental factors and management factors of safety evaluation factors are in the following Table 1 [7].

Table 1 Evaluation Factors

\begin{tabular}{|c|l|}
\hline Evaluation Factor & Safety Index \\
\hline \multirow{3}{*}{$\begin{array}{c}\text { Evaluation Factor } \\
\text { Related to Human }\end{array}$} & Safety Awareness of The Staff \\
\cline { 2 - 2 } & Technical Level of The Staff \\
\cline { 2 - 2 } $\begin{array}{c}\text { Evaluation Factor } \\
\text { Related to Machine }\end{array}$ & Health Status of The Staff \\
\cline { 2 - 2 } Evaluation Factor & The Reliability of Power Equipment \\
\hline Related to Environment & The Level of Security \\
\cline { 2 - 2 } & Normalization of Work Environment \\
\hline \multirow{3}{*}{$\begin{array}{c}\text { Evaluation Factor } \\
\text { Related to Management }\end{array}$} & Embience of Security Culture \\
\cline { 2 - 2 } & Rationality of Staff Allocation \\
\cline { 2 - 2 } & Level of Security Supervision \\
\cline { 2 - 2 } & The Ability of Rectification for Accident Hidden Points \\
\cline { 2 - 2 } & Level of Security Training \\
\hline
\end{tabular}

\section{Comprehensive Evaluation of Electric Power Production Security Based on Diffusible Fuzzy Analytic Hierarchy Process}

Analytic Hierarchy Process Fuzzy Comprehensive Evaluation and Artificial Neural Network method are the main means to evaluate the security of electric power production .Most of these methods are quite subjective and the accuracy is quite limited, without considering the disadvantages of non-linear relationship between every indexes. To solve the above shortcomings, this paper presents a method for evaluation of safety of electricity based on Extension and Fuzzy Analytic Hierarchy Process. According to the index system which has been constructed, the level of safety standards and reviews set can be ensured. The matter-element theory and extension set theory will be made use of and the electric power assessment factors will be quantified. After being quantified, these factors will be express in form of things, features and quantified values. In addition, the fuzzy consistent judgment 
matrix will be confirmed according to Analytic Hierarchy Process with the ascertainment of weight set and calculation of correlation between the factors and electric power safety production, getting the fuzzy membership. Finally, it is feasible to get the assessment grade according to the maximum subordination principle [8-10].

\section{The Main Method of Presenting Information Diffusion and GIS Technology Combined Based on Electricity Safety Early Warning of Venture}

Application of principal component analysis will concentrate the assessment of electricity safety production and early warning of venture indicator system of production safety as the main component of the index system, the use of information diffusion theory will electricity safety production by warning of the risk classification. The method of principal component diffusion index information on the risk of all possible points, which is the main component of the index data input concentrated, and the output is expected early warning signal of the vector[11-13]. Under the early warning of the signal vector, the use of GIS technology and two-dimensional visualization technology for electrical safety risk warning information to show that electrical safety risk intuitive, clearly show to the managers, to facilitate management personnel management decisions, played the purpose of prevention [14].

\section{The Comprehensive Assessment of Electricity Safety Production and Early Warning Integration Platform}

Relying on relevant methods and models proposed in this paper, the use of the Java programming language, based on Struts $2+$ Spring + Hibernate framework research and development of power production safety comprehensive evaluation and integration of early warning platform, and its main function is to achieve power production data acquisition, safety evaluation of power, security situation analysis, safety risk warning, by the power production safety evaluation and risk management of power combined with fully and accurately reflect the overall power production safety level of security and effective early warning of venture, truly dangerous exclude accident before they occur[15].

\section{Conclusion}

Based on the assessment of electricity safety production method and early warning of venture in-depth exploration, using multiple-data acquisition method based on the Service Correlation to collect data, power production collected by using the theory of support vector machine will be classified and stored dynamically; According to the clustering-grey correlation analysis methods to construct the evaluation and risk early-warning index system of safe production of electric power, Using the method of comprehensive safety evaluation of electric power production based on Fuzzy AHP to evaluate in real time; At the same time, warn early by using electric power safety risk early warning method. Based on Main Components Information Diffusion; Finally through the GIS technology and the two-dimensional visualization technology to achieve early warning and evaluation of information management initiative to "jump" eyes, "active" for managers to solve the safety risks of the target. To reduce the probability of accident, to nip in the bud role, optimizing the management of production safety in China, "safety first, safe production and labor protection policy of prevention". To ensure the safe production of electric power, to reduce casualties, to provide users with safe, reliable, qualified electric power has a very important practical significance [16].

\section{Acknowledgements}

This work was supported by Project development plan of science and technology of Jilin Province (No.20140204049GX). 


\section{References}

[1] X. M. Yang: Case Analysis about the Improvement of Enterprise's Electric Power Supply Safety, Technology Wind, (2013) No.18, p. 61. (In Chinese)

[2] An, Z, B: Brief Study on Safety Production Management of Electric Power, Management and Technology of Small and Medium Enterprises, (2011) No.12, p. 16. (In Chinese)

[3] J. Guo: Improving work efficiency of accident prevention of casualty in electric power industry--Interpretation of instruction of electric power casualty accident prevention, Guangxi Electric Power, (2014) No. Z1, p. 61-62. (In Chinese)

[4] B.S. Gai, J. L. Wang and X. Liu: Service-related data sampling methodology, Journal of Xidian University, Vol.40 (2013) No.2, p. 54-59+158. (In Chinese)

[5] L. Qiong, L. Zhang and X. Y. Fang: Establishment of Alarm Systems of Digital Control Room Based on Cluster and Grey Relational Analysis, Journal of University of South China, Vol.28(2014) No.2, p. 11-168.(In Chinese)

[6] G. N. Deng: The Similarity Measure in Clustering, Journal of Northeast Dianli University, Vol. 33(2013) No.1/2, p.156-161. (In Chinese)

[7] Z.Y. Qu, J. Wang, Y. Wu and J.M. Yang: Synthetic Evaluation Method For Electric Power Production Safety Based on the Extension Cloud Model, Safety and Environmental Engineering, Vol.22 (2015) No.3, p. 94-98. (In Chinese)

[8] F. H. Jin and M. J. Zhong: The Training Evaluation of Power Enterprise Based on Fuzzy Hierarchy Comprehensive Evaluation, Journal of Northeast Dianli University, Vol. 33(2013) No.3, p.69-73. (In Chinese)

[9] G. N. Deng: Advances in Fuzzy Reasoning Algorithm, Journal of Northeast Dianli University, Vol. 33(2013) No.6, p.64-70. (In Chinese)

[10]R. J. Song and J. Xu: Establishment and Application of Evaluation Indicator System for Relay Protection Device, Journal of Northeast Dianli University, Vol. 35(2015) No.6, p.70-76. (In Chinese)

[11] J. Lv, Z. A. Jiang and S.F. Wan: Coal enterprise risk based on information diffusion of main components, Journal of China Coal Society, Vol.33 (2008) No.4, p. 477-480. (In Chinese)

[12] J. Zhang, X. D. Ma, X. Liu, S. Y. Liu and Q. Yu: The Evaluation Method of Red Wine Based on Principal Component Analysis, Journal of Northeast Dianli University, Vol.33(2013) No.6, p.41-44. (In Chinese)

[13]X. C. Guo, C. L. Li and X. L. Fan: Research on Text Classification method Based on PCA and KNN, Journal of Northeast Dianli University, Vol. 33(2013) No.6, p.60-63. (In Chinese)

[14]Z.Y. Qu, S. L. Hou, Y. P. Zhang, J. H. Zhang and P. Xin: Realization of Substation Visualization Training Platform, Journal of Northeast Dianli University, Vol.34(2014) No.3, p.75-79. (In Chinese)

[15] C. M. Wu, H. Lin and Z. C. Tian: The Research of Monitoring and Early Warning System of the Wind Power Tower Tilted and Settled, Journal of Northeast Dianli University, Vol.33(2013) No.5, p.22-27. (In Chinese)

[16]D. Z. Lin and Q. Xiao: Sampling and Processing of Electric Power Data, Technology Wind, (2012) No.24, p.50. (In Chinese) 